\title{
Compliance Compliance
}

\author{
Silvina Bacigalupo \\ Universidad Autónoma de Madrid \\ ORCID ID 0000-0001-9619-947X \\ silvina.bacigalupo@uam.es
}

\section{Cita recomendada:}

Bacigalupo, S. (2021). Compliance. Eunomía. Revista en Cultura de la Legalidad, 21, pp. 260-276

doi: https://doi.org/10.20318/eunomia.2021.6348

Recibido / received: 15/10/2020

Aceptado / accepted: 20/07/2021

\begin{abstract}
Resumen
Compliance surge en la discusión jurídica española a partir de la incorporación al Código penal de la responsabilidad de las personas jurídicas en 2010. En 2015, además, se introduce la posibilidad de que los programas de compliance puedan ser una causa de exención de la responsabilidad penal de las personas jurídicas. El término proviene del mundo jurídico anglosajón y se desarrolló en el marco de la autorregulación de las corporaciones privadas. Tras los escándalos políticos y financieros de los años 80' y 90', que dieron lugar a leyes que exigen a las empresas una mayor transparencia y rendición de cuentas, se consolida como herramienta de prevención de sobornos a funcionarios y políticos -caso Watergate-. En la actualidad, sin embargo, ha adquirido una dimensión mayor destinada no solo a la prevención de sobornos, sino a la prevención de los riesgos de la actividad empresarial y delitos que puedan ser cometidos en el marco de la actividad empresarial. Desde una perspectiva integral los programas de compliance son expresión de la cultura empresarial y de una cultura de la legalidad. Tras analizar los distintos aspectos jurídicos inherentes al compliance, se concluye que la cultura empresarial no puede existir sin tener como punto de partida el cumplimiento del Derecho.
\end{abstract}

\section{Palabras clave}

Compliance, cultura empresarial, responsabilidad penal de las personas jurídicas, modelos de compliance, compliance officer, prevención de delitos, prevención de la corrupción, compliance en administraciones públicas.

\begin{abstract}
Compliance originated in the Spanish legal discourse as result of an amendment to the Spanish Criminal Code that introduced the criminal liability of legal persons and corporations in 2010. Furthermore, in 2015, the possibility of compliance programs being caused by an exemption of the criminal liability of legal persons and corporations was introduced. The term comes from Anglo-Saxon law: Following the political and financial scandals of the 80's and 90's, laws were passed that demand more efficacy of private corporations as agencies of prevention of bribes of officials and politicians - see Watergate-could be detected. Currently, however, compliance has assumed a greater dimension destined not only to the prevention of bribes, but also to all crimes that could be committed in the realm of corporate activities in general and as a program of corporate culture and culture of legality. This essay analyzes different legal aspects of
\end{abstract}


compliance and arrives at the conclusion that there cannot be any such thing as a corporate culture if not based on the adherence to the law.

\title{
Keywords
}

Compliance, corporate culture, compliance and criminal liability of corporations, compliance program, compliance officer, prevention of crimes, prevention of corruption, compliance in the public sector.

\begin{abstract}
SUMARIO. 1. Introducción. 2. Antecedentes y significado del término compliance. 3. Compliance y cultura empresarial. 4. Criminal Compliance: Compliance y responsabilidad de las personas jurídicas. 5. Función y elementos de un modelo o programa de compliance. 6 . El deber de implementación de los programas de compliance en la empresa. 7. Función y responsabilidad del Compliance Officer. 8. Compliance y Administraciones Públicas. 9. Conclusiones y resumen.
\end{abstract}

\section{Introducción}

El concepto compliance irrumpe en la discusión científico-penal española a partir de 2010 con la incorporación de la responsabilidad penal de las personas jurídicas en el Código penal (art. 31 bis, introducido por la LO 5/2010). Sin embargo, no es hasta la reforma del Código penal español (LO 1/2015), en que la materia de compliance forma parte de las discusiones académicas y de debate en todos los foros especializados. En este sentido, se ha asentado como una materia inherente a la prevención de delitos en el seno de la actividad empresarial. De ahí que en la actualidad se suela precisar: "compliance penal». La reforma de 2015 incorporó como "causa de exención de la responsabilidad penal» de las personas jurídicas los «modelos de organización y gestión de medidas de vigilancia y control idóneas para prevenir delitos» (arts. 31 bis 2 y $5 \mathrm{CP}$ ). A pesar del término utilizado por el legislador, la terminología que se ha acuñado en la práctica es la del derecho anglosajón y se refiere a ellos como modelos o programas de compliance. Los programas de "cumplimiento normativo» constituyen el sistema interno de control y supervisión de la actividad empresarial mediante el cual las empresas y sus órganos se organizan para operar armónicamente con el Derecho vigente (Hauschka et al., 2010; Rotsch, 2012, p. 2). Se trata de un ámbito de exclusiva autorregulación de normas internas de prevención y disminución de riesgos penales de la actividad empresarial.

Más recientemente, también se ha comenzado a hablar incluso de «compliance en las Administraciones Públicas», entendiendo compliance como una «herramienta» para la prevención de la corrupción en la contratación pública (vid. sobre este tema, infra 8) (Queralt, 2016, p. 1 y ss.).

Antes de centrarnos en qué significa o qué implica el concepto compliance en particular para la gobernanza empresarial y el Derecho penal en la actualidad, es necesario hacer una breve recapitulación de cómo y en qué contexto aparece inicialmente la noción de compliance, cómo se ha llegado a instaurar el compliance en el marco de la actividad empresarial, cómo se encuentra recogido y qué función cumple en el Código penal y los distintos efectos que tiene en la actualidad en el marco de la responsabilidad penal de las personas jurídicas. 


\section{Antecedentes y significado del término compliance}

El término "compliance» proviene, como ya se ha dicho, del derecho anglosajón y significa "cumplimiento», "obediencia», "respeto», entro los primeros significados del término. En el ámbito jurídico se identifica con el "cumplimiento normativo» y desde el punto de vista de la estructura empresarial se refiere de forma más extensa a la "organización de las empresas para el desarrollo de la actividad empresarial conforme a derecho». Por regla general, se refiere de forma específica al cumplimiento de normas legales, pero también a normativa o políticas internas y estándares de buenas prácticas o de soft law. También encontraremos, en este contexto, el uso del término "compliance regulatorio» (regultarory compliance) (Lin, 2017 , p. 159). En este sentido, compliance surge y se utiliza en el contexto del «marco de autorregulación» y de la libertad para decidir la organización interna de las sociedades mercantiles de Derecho privado orientada a la prevención de los riesgos provenientes de su actividad empresarial.

En sus comienzos, no obstante, no hay que dejar de tener en cuenta que el origen de compliance moderno se forja a inicios del siglo XX en EE.UU. con la creación de las primeras «agencias públicas de regulación». En particular, con la creación de las «Agencias Públicas de Seguridad en Estados Unidos» -p.ej. Food and Drug Administration, 1906, FDA ${ }^{1}$-. Es decir, surge en el ámbito del «derecho regulatorio de los primeros sistemas de supervisión pública regulada».

En el marco de la actividad empresarial en EE.UU. el término compliance surge vinculado al marco de las «normas de soft law» y, en particular, a «recomendaciones de buenas prácticas» basadas en el principio "complain or explain» («cumplir o explicar»). Se trata de normas o recomendaciones de "cumplimiento voluntario» que son establecidas por organismos u organizaciones gubernamentales y no gubernamentales con la finalidad de que, en el ámbito del sector privado, las empresas incorporen las recomendaciones establecidas o, en caso de no hacerlo, expliquen la razón o razones que las lleva a no seguir dichas recomendaciones. En este ámbito se trataba de la incorporación de «recomendaciones de buena gobernanza, de rendición de cuentas y de responsabilidad social corporativa (RSC)».

En los años '70 y ' 80 las normas de compliance de la actividad empresarial evolucionan en otro escenario: los sonados escándalos de corrupción política, sobornos y financiación de los partidos políticos que afectaron a importantes compañías y que alcanzaron su máxima expresión con el caso Watergate. Como consecuencia de aquel escándalo, se dictó en EE.UU. la Foreign Corrupt Practices Act (1977), que incorporó requerimientos y prohibiciones para las empresas y el sector privado en materia de sobornos, libros y registros, así como, por primera vez, la figura de compliance, obligando a la transparencia de las cuentas anuales y prevención de la corrupción de funcionarios.

En los años '90 se evidencian nuevos riesgos de la actividad empresarial vinculados a los escándalos financieros -los casos Enron, Tyco International, WorldCom, Siemens- que dieron lugar, a su vez, a la Sarbanes-Oxley-Act (2002), por la que se establecen nuevas obligaciones en materia contable y se reforman las exigencias de contabilidad pública de las empresas y protección del inversionista.

\footnotetext{
${ }^{1}$ La Food and Drug Act (FDA) fue la primera norma que regulaba y establecía estándares para la protección de los consumidores, cuyo incumplimiento conllevaba sanciones y la supervisión estaba atribuida a una agencia pública de supervisión.
} 
Los sobornos en las transacciones económicas transnacionales de las empresas y los escándalos financieros acotaron un primer escenario para el compliance de la actividad empresarial: corrupción y soborno de funcionarios, financiación de la política, maquillajes de la contabilidad y falta de transparencia.

Conscientes de este escenario los organismos internacionales -OCDE, UNy supranacionales -UE- comienzan a elaborar convenios para la prevención de la corrupción. Se desarrollan el Convenio para combatir el cohecho de servidores públicos extranjeros en transacciones comerciales internacionales (OCDE 1997) ${ }^{2}$, el Convenio penal contra la corrupción (Convenio $n^{\circ} 173$ del Consejo de Europa) $(1999)^{3}$, la Convención de las Naciones Unidas contra la corrupción (2004)4. En este mismo contexto, se impulsa también la UK Bribery Act (2010) que introduce, a su vez, la responsabilidad penal de las empresas y de sus directivos por sobornos a funcionarios extranjeros y exigencias de órganos de supervisión y control. Todas estas normas imponen a los Estados el compromiso de establecer normas para la prevención y supervisión de los riesgos de corrupción en la actividad empresarial que dieron lugar a importantes cambios legislativos en el ámbito del mercantil, administrativo y penal de la actividad empresarial.

Se puede ver como la evolución del término "compliance» evoluciona en relación a la actividad empresarial y la cultura jurídica de corte anglosajón con la finalidad principal dirigida al establecimiento de «mecanismos internos de control y supervisión» vinculada, inicialmente, a la prevención de la corrupción, los sobornos a funcionarios públicos y la financiación ilegal de partidos políticos.

Paralelamente, a mediados de los '90 los casos mencionados de empresas vinculadas a los escándalos contables y la crisis financiera ponen de manifiesto el escaso control que existe de los procesos de adopción de decisiones por parte de los órganos de administración de la empresa. La organización de los órganos de administración estaba regulada de forma extraordinariamente amplia, dejando un marco de autoorganización de la gestión empresarial con todo el poder de decisión y ausencia de control y supervisión de las actuaciones del mismo ${ }^{5}$. Se abre así otra exigencia: transparencia en la "gobernanza corporativa» (corporate governance). Ello da lugar a una importante revisión del derecho de las sociedades mercantiles y, en particular, a la incorporación de estándares de buenas prácticas a través de los "Códigos de buenas prácticas o Códigos de Buen Gobierno Corporativo» para las sociedades cotizadas. Es aquí donde surgen de forma patente las primeras recomendaciones de organización, supervisión y control de la actividad empresarial que, en última instancia, forma parte también de un programa de compliance. Los primeros informes que advertían en España esta problemática y estudiaron las medidas de buen gobierno necesarias en el marco de las sociedades cotizadas fueron los Informe de la Comisión especial para el estudio de un código ético de los consejos de administración de las sociedades (Comisión Olivencia, 1998) y el Informe de la comisión especial para la transparencia y la seguridad de los mercados financieros y

\footnotetext{
${ }^{2}$ Convención para combatir el cohecho de servidores públicos extranjeros en transacciones comerciales internacionales y documentos relacionados, recuperado de https://www.oecd.org/daf/antibribery/ConvCombatBribery Spanish.pdf

${ }^{3}$ Instrumento de Ratificación del Convenio penal sobre la corrupción (Convenio número 173 del Consejo de Europa) hecho en Estrasburgo el 27 de enero de 1999, recuperado de https://www.boe.es/diario boe/txt.php?id=BOE-A-2010-12135.

4 Convención de las Naciones Unidas contra la Corrupción, Viena, 2004, recuperada de https://www.unodc.org/documents/treaties/UNCAC/Publications/Convention/04-56163 S.pdf

${ }^{5}$ Basta hacer un repaso desde la vieja Ley de Sociedades Anónimas hasta la actual Ley de Sociedades de Capital para ver la progresiva delimitación de los deberes y obligaciones de los órganos de administración.
} 
sociedades cotizadas (Comisión Aldama, 2003) ${ }^{6}$. En 2006 la Comisión Nacional del Mercado de Valores (CNMV) incorporó el Código Unificado de Buen Gobierno de las Sociedades Cotizadas (el llamado, Código Conthe, 2006) y, actualmente, el vigente Código de Buen Gobierno de las Sociedades Cotizadas (2013, 2015, 2020). El CBG contiene las recomendaciones exigibles a las sociedades cotizas, así como la exigencia de presentación de un Informe Anual de Buen gobierno -propuesto por el Informe Aldama-. Finalmente, la reforma de la Ley de Sociedades de Capital en 2014 convirtió en obligación legal muchas de las recomendaciones del Código de Buen Gobierno, en particular, en relación a la organización del gobierno corporativo estructura del consejo de administración, comisiones delegadas, distinción de los deberes de supervisión, etc., arts. 212 ss. LSC-, los deberes de administradores deberes de lealtad, diligencia, secreto, etc., arts. 225 y ss. LSC- y, sobre todo, para las cotizadas los deberes indelegables del Consejo de administración, entre los que se incluye la determinación de la política de control y gestión de riesgos (art. 529 ter b) LSC) o la política de responsabilidad social corporativa (art. 529 ter a) LSC).

Finalmente, en el ordenamiento jurídico español se introduce o, mejor dicho, se menciona de forma expresa, como hemos señalado, a partir de la reforma del Código penal (LO 1/2015, de 30 de marzo) al incluirlo el legislador como una «causa de exención de responsabilidad penal de las personas jurídicas». El Código penal se refiere a los programas de compliance con la denominación de «modelos de organización y gestión de medidas de vigilancia y control idóneas para prevenir delitos» (art. 31 bis $2,1^{\circ}$, y $5 \mathrm{CP}$ ). Es decir, los programas de cumplimiento se introducen de forma expresa en un ámbito muy concreto y delimitado referido a «medidas de prevención de delitos en el ámbito de las empresas y otras personas jurídicas» (de acuerdo con el art. 35 Código civil, entre otras, asociaciones, fundaciones, partidos políticos y sindicatos). Sin embargo, con anterioridad ya existía normativa sectorial -en el ámbito del sector financiero, bancario y del mercado de valores- que establecían normas expresas de organización para la supervisión de riesgos de la actividad empresarial en relación con una determinada actividad de negocio ${ }^{7}$ (vid. infra).

En la actualidad, se puede decir que la institución de la figura de compliance ha sido consecuencia, al menos, de dos causas: en primer lugar, de la ausencia o escasa existencia de normas reguladoras de la organización interna de las corporaciones privadas $y$, en segundo lugar, también de la insuficiencia de recursos gubernamentales para un adecuado modelo de supervisión pública de los riesgos de la actividad empresarial centralizada. Como consecuencia de ello los «modelos de supervisión» se han visto «privatizados», en el sentido, de trasladar a los particulares y a las empresas un «deber de autorregulación» de la supervisión y control del

\footnotetext{
${ }^{6}$ Véase toda la documentación, referencias legislativas e Informes de Buen Gobierno, en: www.cnmv.es y https://www.cnmv.es/portal/Legislacion/COBG/COBG.aspx

${ }_{7}^{7}$ Véase, por ejemplo, Circular CNMV n 6/2009, de 9 diciembre, sobre control interno de las sociedades gestoras de instituciones de inversión colectivas y sociedades de inversión, Circular CNMV n 1/2014, de 26 de febrero, sobre los requisitos de organización interna y de las funciones de control de entidades que prestan servicios de inversión, Código de Buen Gobierno de las Sociedades Cotizadas, 24 febrero 2015, Principio 21: «la sociedad dispondrá de una función de control y gestión de riesgos ejercida por una unidad o departamento interno, bajo supervisión directa de la comisión de auditoría o, en su caso, de otra comisión especializada del Consejo de administración», RD 304/2014, de 5 de mayo, Reglamento de la Ley 10/2010 de Prevención de blanqueo de capitales (Cap. IV: procedimientos de control, análisis de riesgos, contenido mínimo del Manual de prevención, órganos y medidas de control, obligaciones de formación de empleados y directivos), o la Ley 31/2014, de 3 de diciembre, por la que se modifica la Ley de Sociedades de Capital para la mejora del gobierno corporativo.
} 
cumplimiento normativo y prevención de incumplimiento, llevando el cumplimiento normativo más allá del ámbito de la propia función de la supervisión pública ${ }^{8}$.

Todo este recorrido permite ver, en primer lugar, que la noción de compliance surge en el contexto de sociedades mercantiles de "derecho privado imponiendo", en el marco de su libertad de autorregulación, exigencias de «control y supervisión de los riesgos derivados de su actividad»; y, en segundo lugar, surge como consecuencia de la necesidad de exigir a las sociedades mercantiles «mayores estándares de organización interna» de los órganos de administración y adopción de decisiones para poder detectar eventuales riesgos de corrupción y lograr una mayor transparencia de la gestión -caso Watergate-. En tercer lugar, las exigencias de compliance se refuerzan como consecuencia de los fraudes financieros y la crisis económica y financiera -casos Enron, Parmalat, Tyco International, WorldCom, Siemens, Lehman Brothers, Subprime etc.- y pasa de ser, en una primera instancia, un conjunto de normas de soft law de buen gobierno corporativo -Códigos de Buen gobierno corporativo- para convertirse rápidamente en «normas legales de obligado cumplimiento» para la gobernanza de las sociedades, deberes legales de supervisión y controles internos de riesgos en el marco de la actividad empresarial que abarcan en la actualidad deberes y riesgos desde el Derecho mercantil, Derecho tributario, Derecho laboral, Derecho administrativo hasta llegar al Derecho penal.

Es escenario de riesgos de la actividad empresarial y la incorporación de la responsabilidad penal de las personas jurídicas coloca al compliance y a la «cultura empresarial de los negocios» como pilares esenciales de la organización y de la gobernanza corporativa (Bacigalupo, 2011, pp. 17 y ss.) ${ }^{9}$.

\section{Compliance y cultura empresarial}

El ámbito de compliance no solo debe circunscribirse al cumplimiento normativo para la prevención de riesgos de gobernanza y de delitos, como actualmente se ha consolidado. Teniendo en cuenta el complejo escenario normativo de soft y hard law, compliance debe ser entendido de forma integral y transversal como un "paraguas» de la organización empresarial: constituye el marco de referencia de la «cultura de valores» de una empresa -cultura empresarial- (Schein, 1986, pp. 677 y ss.). En este sentido, resulta evidente que no hay ninguna cultura que pueda fundamentarse en el incumplimiento legal, por lo que el «cumplimiento normativo debe ser la base de toda cultura empresarial».

En la literatura anglosajona se define «cultura empresarial» de forma amplia como «un activo intangible cuya función es facilitar la gestión de las contingencias imprevistas» (Kreps, 1990, pp. 90 y ss.) e incluye «las asunciones, valores y creencias comunes a los que forman la empresa y que ayudan a los empleados a saber qué tienen que hacer» (Schein, 1986, pp. 677 y ss.) cuando dichas contingencias imprevistas acaecen.

La cultura empresarial se constituye, en definitiva, sobre los valores con los que una empresa se relaciona con el mundo circundante, por un lado, con sus

\footnotetext{
${ }^{8}$ En este sentido, un claro ejemplo son las normas de prevención del blanqueo de capitales que han trasladado a los particulares (a entidades bancarias, a los abogados, a los notarios, a los intermediarios financieros, etc.) el control y la supervisión de actividades sospechosas de blanqueo de capitales; dichos sujetos obligados son los que deben hacer la primera supervisión y trasladar a las autoridades públicas actividades sospechosas.

${ }^{9}$ Cfr. Alfaro (2016).
} 
stakeholders (Grennan, 2019), pero, sobre todo, supone la «definición de la empresa como sujeto y su relación con la sociedad en su conjunto» ${ }^{10}$.

Se puede apreciar cómo no es solo una cuestión de «cultura, principios o valores», sino que ha de traducirse en pautas de actuación que puedan guiar el correcto proceder en la actividad empresarial. De aquí que podamos extraer al menos dos consecuencias: a) desde un punto de vista legal, compliance no solo es prevención de riesgos penales, sino cumplimiento y prevención de todas las normas civiles, administrativas y sectoriales que rigen la actividad empresarial y de obligado cumplimiento según la actividad de cada empresa, y b) desde un punto de vista de cultura empresarial la empresa podrá incorporar cuantas normas de soft law considere parte determinar «sus valores de actuación»: aquí entrarían todo el conjunto de recomendaciones de organismos gubernamentales y no gubernamentales, organismos internacionales, etc. -recomendaciones de UN, OCDE, Transparency International, etc.-

En este sentido, el cumplimiento del Derecho es el marco y el techo de las exigencias legales y, por tanto, no pueden existir más consecuencias sancionadoras que las dispuestas en las normas jurídicas y que se deriven del incumplimiento de las mismas (art. $25 \mathrm{CE}$ ). El incumplimiento de normas de soft law, autoimpuestas como cultura empresarial, solo podrá tener las consecuencias que se les depare en un "código de conducta»-o código ético- de forma expresa y enmarcadas, en todo caso, en el ámbito de las sanciones posibles que permita el Derecho laboral. Sin duda que el incumplimiento de las normas autoimpuestas tendrá repercusiones reputacionales en el mercado y en los inversores. Dicho con otras palabras, a los efectos normativos los valores de la cultura empresarial deben ser traducidos a «normas de conducta» concretas recogidas en el código de conducta/ético, como norma fundamental de la actividad empresarial basada en valores y las sanciones por su incumplimiento solo podrá estar enmarcado en el contexto de las sanciones posibles de las infracciones al derecho laboral -fundamentalmente, infracción del deber de buena fe contractual. En este sentido, el código de conducta o código ético de una empresa es el instrumento normativo en el que se plasma la cultura empresarial basada en valores y los principios rectores de la empresa como sujeto.

En este sentido, las empresas tienden a incorporar a sus códigos de conducta los más altos estándares de buenas prácticas estableciendo severas exigencias que de no estar formuladas con precisión, dejando claro la conducta exigida a los destinatarios de las normas, pueden llegar a dificultar el correcto cumplimiento del comportamiento empresarial esperado. Incluso, se puede apreciar que cuanto más alto sea el estándar de diligencia que se fije la propia empresa, ésta podría llegar a incurrir en autorregularse en riesgos penales (Kuhlen, 2009, p. 11 y pp. 26 y ss.; Hauschka y Greeve, 2007, p. 165).

Una cultura empresarial basada en el cumplimiento normativo cumple con los valores de una sociedad, pues implican el respeto de las normas constitucionales, de las normas que limitan los comportamientos que la sociedad no tolera -es decir, las conductas previstas como delitos en el Código penal-, de las normas que rigen el mercado y la libre concurrencia en el mercado, de las normas que protegen a los trabajadores y a los consumidores, y así podríamos seguir con todas las normas legítimamente emanadas de las instituciones que en definitiva son el marco de los

\footnotetext{
${ }^{10}$ Confr. extensamente sobre la configuración del sujeto Bacigalupo, S. (1998, pp. 103 y ss. [Capítulo II: «La idea de sociedad y el concepto del sujeto en la sociología y la ética material como fundamento de la dogmática»]; Luhmann, 1995, pp. 124 y ss.).
} 
valores de una sociedad en la que impera el Estado de Derecho (Bacigalupo, 2011, pp. 18 y ss.).

En resumen, «cultura empresarial es o debe ser, ante todo, cultura de la legalidad».

Desde esta perspectiva un «programa de cumplimiento normativo» debe incluir el respeto de las normas jurídicas y la definición de los valores de la actividad empresarial que pueden incorporar el amplio elenco existente de normas de voluntario cumplimiento como compromiso de una actividad empresarial basada en los máximos estándares de buen gobierno corporativo.

\section{Criminal Compliance: Compliance y responsabilidad penal de las personas jurídicas}

Como hemos adelantado en la introducción, tras la reforma del Código penal (LO $1 / 2015$, de marzo), se incorporan los «modelos de organización y gestión» inspirados en los modelos de compliance estadounidenses como una «causa de exención de la responsabilidad penal»-véase, regulación general de las causas que eximen de la responsabilidad criminal, Libro I, Capítulo II, arts. 19-20 CP-. De acuerdo con lo dispuesto en el Código penal, los «modelos de organización y gestión» para poder ser una causa eximente de la responsabilidad penal deben incluir «medidas de vigilancia y control idóneas para prevenir delitos de la misma naturaleza o para reducir de forma significativa el riesgo de su comisión» (art. 31 bis 2. $1^{\text {a }} \mathrm{CP}$ ) y deben establecer un «órgano en la persona jurídica con poderes autónomos de iniciativa y control para la supervisión del funcionamiento y del cumplimiento del modelo de prevención» (art. 31 bis 2. $2^{\mathrm{a}} \mathrm{CP}$ ). Por último, señala el Código penal que éstos podrán tener el efecto eximente «siempre que no se haya producido una omisión o un ejercicio insuficiente de las funciones de supervisión, vigilancia y control por parte del órgano» (art. 31 bis $2.4^{\mathrm{a}}$ ) o los autores individuales hayan cometido el delito eludiendo fraudulentamente los modelos de organización y prevención (art. 31 bis 2, $3^{\text {a }} \mathrm{CP}$ ).

Es preciso señalar y dejar claro que el Código penal no impone a las empresas una obligación legal de implementar un modelo de prevención de delitos. Solo establece las condiciones bajo las cuales, llegado el caso, las personas jurídicas podrían quedar exentas de responsabilidad penal. Por lo tanto, no establece ninguna posición de garante de los miembros del consejo de administración para implementar estos modelos de prevención de delitos. Por el contrario, los deberes de los miembros de un consejo de administración se encuentran dispuestos en los arts. 225 y ss. de la LSC. Son estos deberes de los administradores dispuestos en la LSC los que configuran la «posición garante» del órgano de administración y, por tanto, pudieran dar lugar a la responsabilidad de los administradores por omisión de la implementación de los programas de compliance si llegara a producirse algún delito en el marco de la actividad empresarial (art. 11 CP) (Bacgalupo, 2011, pp. 45 y ss.; Ruiz de Lara et al., 2018, pp. 22 y ss.). Por lo tanto, la eventual falta de implementación de los modelos de prevención de riesgos por parte del Consejo de administración podría llegar a producir un delito de administración desleal (art. 252 CP) si, como consecuencia de un delito imputable a la empresa, se derivara algún perjuicio para el patrimonio social. Dicho con otras palabras: el Código penal no establece el deber de implementación de estos modelos, pero si su falta de implementación llegara a producir un perjuicio económico a la sociedad no habría que excluir que el órgano de administración pudiera haber cometido un delito de administración desleal. Por lo tanto, en aras al cumplimiento del deber de diligencia (art. 225 LSC) y la posición de garante que éste genera para todo administrador de velar por la evitación de riesgos 
a la sociedad, una actuación diligente de los administradores es adoptar los modelos de prevención de riesgos penales (vid. más extensamente infra, 7).

Los modelos de prevención del art. 31 bis del CP solo están contemplados como causa eximente de la responsabilidad penal de las personas jurídicas, por lo que como tal no eximen de responsabilidad a la persona física que actuó. Se trata de poder constar hasta qué punto un programa de organización y control de riesgos ha sido capaz de "reducir» la concreción de los riesgos penales y ello pueda servir de causa de exención o reducción de la responsabilidad penal de la persona jurídica (Bock, 2009, pp. 68 y ss.).

En este contexto, compliance adquiere una dimensión y peculiaridades específicas que permite, sin duda, concretar su sentido y contenido conceptual como Criminal Compliance (Rotsch, 2012, p. 2). No obstante, aún son escasos los trabajos académicos que han abordado de forma pormenorizada qué supone el fenómeno del compliance desde un punto de vista político-criminal y dogmático (Bock, 2011).

\section{Función y elementos de un modelo/programa de compliance}

Un programa de compliance o modelo de gestión y prevención de riesgos penales, como su propio nombre indica, tiene la finalidad de prevenir y disminuir de forma considerable la posibilidad de comisión de delitos en el marco de la actividad empresarial.

Para poder cumplir con dicho objetivo un programa de cumplimiento consta de cuatro elementos esenciales a las que el Código penal hace referencia en su art. 31 bis 5: medidas de prevención, medidas de detección, medidas de reacción y medidas de seguimiento. A su vez, cada una de estas fases se componen de diversos "elementos» (Bacigalupo, 2011, passim; Kuhlen et al., 2013, passim; de forma extensa, Aguilera Gordillo, 2018, pp. 257 y ss.):

Fase 1: Prevención. Poder prevenir los riesgos de la actividad empresarial requiere conocer los riesgos de la propia actividad empresarial. Por ello, se requiere como primera actividad la necesaria elaboración de un «mapa de riesgos» (art. 31 bis 5. $1^{\text {a }} \mathrm{CP}$ ). La identificación y el análisis de los riesgos penales de la actividad empresarial permitirá establecer las medidas necesarias y previsibles que pudieran eliminar o disminuir considerablemente dichos riesgos. El establecimiento de las medidas tendentes a la reducción de los riesgos penales, se deben establecer por escrito en los llamados "protocolos internos» o políticas internas que determinen en la estructura empresarial los distintos roles -o puestos-, las distintas funciones y mecanismos de toma de decisiones, los deberes de control, supervisión e información, etc. (art. 31 bis $5.2^{\mathrm{a}}$ ). Estos son los dos elementos principales de la una estructura de prevención -análisis de riesgos y medidas de prevención-, pero para ser eficaces requieren ser conocidas por todos los empleados, que éstos hayan recibido la correcta formación para saber detectar los riesgos y cumplir de forma adecuada con los deberes de control y supervisión.

En resumidas cuentas, la fase de prevención consta de: mapa de riegos matriz de riesgos- de la actividad empresarial, protocolos internos -código de conducta, protocolos de políticas internas y procedimientos-, integración de los procesos, centralización de la función del compliance officer-supervisión y control-.

Fase 2: Detección. La fase de detección es la fase en que se deben llevar a cabo los deberes de control y supervisión del correcto funcionamiento de los protocolos internos. Para poder realizar la esta función es necesario disponer de 
adecuados canales de comunicación entre el compliance officer y los demás empleados en la empresa. Entre otros medios, se entiende que es necesario contar con los denominados "canales de denuncias internas" -whistleblower (Bacigalupo, 2021, p. 163 y ss. ) - a fin de facilitar el conocimiento por parte del compliance officer de las eventuales infracciones de los protocolos que se puedan cometer por los empleados y directivos. Asimismo, para la prevención de determinados delitos, como, por ejemplo, los de corrupción en los negocios, se considera que es esencial contar un canal de denuncias externo. Se discute en la actualidad si las denuncias deben ser anónimas o si es suficiente con que éstas sean confidenciales. La denuncia anónima tiene un principal problema práctico a efectos del eventual procedimiento penal que pudiera dar lugar un incumplimiento, la ausencia de un testigo principal. Por otro lado, las denuncias anónimas pueden ser un medio que propicie denuncias falsas o basadas en sospechas no acreditables. La virtud fundamental que ven los partidarios de las denuncias anónimas basadas en el modelo anglosajón es la posibilidad que brinda el anonimato para proteger a los denunciantes de eventuales represalias internas en el lugar de trabajo. La denuncia confidencial, en principio, debería poder garantizar el anonimato inicial del denunciante, si se establecen adecuadamente los mecanismos de protección. La doctrina mayoritaria y las propuestas legislativas, tanto a nivel nacional como europeo e internacional, así como las recomendaciones de las organizaciones internacionales, abogan por las denuncias anónimas basadas en el modelo anglosajón. En todo caso, es preciso señalar que la recepción de una denuncia, aún en un modelo de denuncias confidenciales, no implica que ésta no se pueda investigar. Al contrario, sería suficiente como notitia criminis y permitiría, de ser verosímil, iniciar al menos unas primeras diligencias de investigación. Sean anónimas o confidenciales las denuncias internas, en todo caso, la empresa debe garantizarse la no existencia de represalia alguna como consecuencia de que un trabajador haya interpuesto una denuncia.

Fase 3: Reacción. Una vez detectado un incumplimiento o infracción del código de conducta o de los protocolos internos o recibida una denuncia, el departamento de compliance debe iniciar una investigación interna a fin de valorar y resolver la infracción. Como toda investigación de una infracción, también las investigaciones internas, están sometidas al cumplimiento de los derechos de los trabajadores, a las garantías constitucionales de todo procedimiento sancionador -a la presunción de inocencia, derecho de defensa, procedimiento contradictorio, principio de culpabilidad e in dubio pro reo, etc.-. Una vez concluida la investigación interna y en caso de poderse afirmar la existencia de una infracción, es necesario que tal infracción conlleve una consecuencia disciplinaria. El sistema disciplinario en las relaciones laborales está expresamente previsto en la legislación laboral-Estatuto de los Trabajadores, convenios colectivos, etc.-. Por lo tanto, en este contexto no se trata de que cada empresa tenga un sistema disciplinario autónomo, sino que haga aplicar las normas sancionadoras previstas en la legislación. En esta misma fase muchas veces pudiera aflorar que los controles de prevención establecidos no han sido suficiente y/o deberían ser reforzados. Por lo tanto, permite revisar y actualizar las medidas de prevención.

Fase 4: Seguimiento. Por último, el cuarto y último pilar de un programa de compliance es el seguimiento, verificación y actualización del programa de prevención. Un programa de cumplimiento no es estático, sino que requiere de actualización permanente. El Código penal ofrece los «criterios esenciales para la verificación» periódica y/o eventual modificación de los procesos de control (art. 31 bis $5.6^{\circ} \mathrm{CP}$ ): cuando se pongan de manifiesto infracciones relevantes de las disposiciones del programa, cuando se produzcan cambios en la organización, en la estructura de control o en la actividad desarrollada. El hecho de que no se hayan puesto de manifiesto casos de infracción no debe llevar a la errónea conclusión de 
que el programa funciona. Puede ser que los controles no sean lo suficientemente rigurosos y, por ello, no permitan detectar fallos. De ahí que la revisión y seguimiento es un proceso constante. La Circular de la FGE 1/2016, incluso, indica que lo razonable es una revisión experta externa cada mínimo dos años. Tomo como referencia las normas de auditorías.

Por último, conviene tener presente que un «programa de compliance» no es ni puede ser un programa estándar, ni siquiera en cuanto a una misma actividad -así también, lo señalan correctamente las Circulares de la FGE 1/2010 y 1/2016-. Lo único estándar -al menos de momento y con el estado de conocimiento actual- es la «metodología» -es decir, las cuatro fases: prevención, detección, reacción, seguimiento-y los «indicadores de riesgos generales de carácter sectorial» recogidos en la legislación sectorial, como, por ejemplo, la referente a la prevención del blanqueo de capitales. A partir de ahí, el modelo de prevención de riesgos depende esencialmente de la actividad empresarial concreta que desarrolle en el sector, del tamaño de la corporación, de su estructura orgánica, de los distintos procesos internos, entre otros. Ello implica, además, que no se trate una mera labor de auditoría de procesos internos de la empresa, sino de un diseño jurídico -con especial énfasis en los aspectos jurídico-penales en cuanto a los mecanismos de prevención de delitos- de distribución de posiciones de garante -obligaciones de supervisión y control- en los distintos niveles de toma de decisiones internas, hasta de las más esenciales, para poder determinar los procesos de formación de decisiones.

\section{El deber de implementación de los programas de compliance en la empresa}

En la empresa la administración de la sociedad reside en los administradores y sus deberes y obligaciones están dispuestos en la Ley de Sociedades de Capital -R-DL 1/2010, de 2 de julio, Texto refundido de la Ley de Sociedades de Capital, Título VI, arts. 209 y ss.-. En particular, el art. 225 LSC establece el «deber de diligencia», como deber principal, de los administradores. Se trata de una conceptualización y desarrollo del viejo conocido "deber del ordenado empresario», al que aún hace referencia el actual art. 225 LSC. El deber de diligencia se complementa además con funciones indelegables del consejo de administración -art. 249 bis LSC; y, con más funciones indelegables, art. 528 ter si se trata de sociedades cotizadas-. Se trata de funciones -deberes jurídicos- que atribuyen a los administradores la posición de garante de la cual pueden derivarse las eventuales responsabilidades jurídicas por incumplimiento normativo.

Es de esta posición de garante basada en el deber de diligencia que surge la obligación, en el marco de una correcta gestión, el deber de implementación de los programas de compliance en el marco de la sociedad (Bacigalupo, 2011, pp. 79 y ss.; Silva Sánchez, 2013, pp. 71 y ss.). Como ya hemos señalado, el Código penal no establece la obligación de implementación de los programas de compliance -en el proyecto de reforma de 2014 se recogía esta figura como delito de omisión de la implementación de los modelos de organización y control, art. 286 bis, castigado con una pena muy leve, que finalmente no fue implementado-. El Código penal solo establece que, en caso de un delito que fuera imputable a la empresa, ésta podría llegar a quedar exenta de responsabilidad si hubiese tenido con anterioridad a la comisión del delito un programa de compliance que hubiese podido evitar o disminuir el riesgo (art. 31 bis CP). No hay responsabilidad penal de los administradores por la no implementación de programas de compliance. Por tanto, dicho deber jurídico solo puede surgir del «deber general de diligencia» que implica cumplir con la diligencia de un ordenado empresario. En este sentido, como ya hemos señalado, un sector minoritario en la doctrina sostiene que los perjuicios patrimoniales que la falta de 
existencia de un programa de compliance pudiera acarrear a la sociedad, pudieran dar lugar a un delito de administración desleal (art. 252 CP).

Sin embargo, es preciso no olvidar que existen exigencias de implementación de figuras especiales como la del compliance officer previamente en el marco de «normativa sectorial específica», como en la Circular CNMV $n^{\circ}$ 6/2009, de 9 diciembre, sobre control interno de las sociedades gestoras de instituciones de inversión colectivas y sociedades de inversión, Circular CNMV nº 1/2014, de 26 de febrero, sobre los requisitos de organización interna y de las funciones de control de entidades que prestan servicios de inversión, Código de Buen Gobierno de las Sociedades Cotizadas, 24 febrero 2015, Principio 21: «la sociedad dispondrá de una función de control y gestión de riesgos ejercida por una unidad o departamento interno, bajo supervisión directa de la comisión de auditoría o, en su caso, de otra comisión especializada del Consejo de administración», RD 304/2014, de 5 de mayo, Reglamento de la Ley 10/2010 de Prevención de blanqueo de capitales -Cap. IV: procedimientos de control, análisis de riesgos, contenido mínimo del Manual de prevención, órganos y medidas de control, obligaciones de formación de empleados y directivos-, Ley $31 / 2014$, de 3 de diciembre, por la que se modifica la Ley de Sociedades de Capital para la mejora del gobierno corporativo.

\section{Función y responsabilidad del Compliance Officer}

La función del compliance officer u «oficial de cumplimiento» es velar por el cumplimiento de la legalidad, por el cumplimiento de las normas internas y del ordenamiento jurídico en el marco de la actividad empresarial (Bacigalupo, 2020; Turienzo Fernández, 2021, passim). Dicho con otras palabras, su «función es de control y supervisión», es una actividad que incluye tanto funciones de intervención control jurídico-, como de auditoría -supervisión de procesos-, de consulta previa para evitar la vulneración de protocolos internos, de formación de los empleados en el correcto conocimiento del programa y las políticas internas, etc.

En este contexto, se discute si el compliance officer tiene que ser un profesional con formación jurídica o puede tener cualquier otro tipo de formación superior -economista, auditor, ingeniero, etc.-. En la medida en que se trata de una función de control y supervisión de cumplimiento «normativo» parece necesario que quien desempeñe dicha función tenga conocimientos jurídicos. Existen, no obstante, muchas opiniones que difieren de esta y consideran que puede ser una persona con cualquier otro tipo de formación. Resulta difícil imaginar que para la construcción de un puente se contratara a un ingeniero. Lo que es claro, es que en la función que debe desempeñar un departamento de compliance sería recomendable que fuera trasversal y se pudiera tener un auditor, un ingeniero informático y un jurista.

Desde un punto de vista estrictamente jurídico-penal el compliance officer se encuentra en posición de garante como cualquier otra persona en la empresa (art. 11 $\mathrm{CP}$ ) y puede responder también penalmente por omisión: por ausencia de control o control insuficiente, o bien también por no investigar cuando detecta un incumplimiento. El compliance officer puede responder por todas las categorías de imputación del Código penal -autoría, coautoría, cómplice o, incluso, como autor de un delito de encubrimiento (art. 451 ss. CP)-.

En la medida en que el «deber general de diligencia» (art. 209 LSC) reside en el Consejo de administración, desde un punto de vista estrictamente societario, el compliance officer vendría a ocupar una «función delegada del Consejo de administración» (art. 529 terdecies LSC) en la «prevención, control y supervisión de cumplimiento normativo» en toda la empresa. Esta función no está expresamente 
prevista en la legislación mercantil, como, por el contrario, sí lo está la comisión de auditoría (art. 529 quaterdecies LSC). De existir un lugar en el cual habría que regular las funciones del compliance officer esta sería en el marco de las comisiones delegadas o funciones delegadas del órgano de administración.

Aunque aún no se ha discutido en profundidad, un tema que en la práctica está adquiriendo cierta relevancia es si las funciones del compliance officer abarcan también el control y supervisión de las acciones del consejo. En mi opinión, no. Velar porque las actuaciones del consejo de administración se ajusten a la normativa aplicable y sean conforme a los estatutos del consejo y demás normativa aplicable, es decir, sean conforme a derecho, es una de las funciones del secretario del consejo de administración (art. 529 octies LSC). Dicho con otras palabras: el compliance officer de cumplimiento normativo del consejo de administración es el secretario del consejo.

\section{Compliance y Administraciones Públicas}

En la actualidad, se ha abierto un nuevo ámbito de debate sobre la posibilidad de trasladar la idea de compliance a las Administraciones públicas como mecanismo tendente a la prevención de la comisión de delitos vinculados a los casos de corrupción en la contratación pública. Se ha planteado por parte de algún sector doctrinal (Queralt, 2016, pp. 1 y ss.) la idea de incorporar la idea de public compliance y la figura de un public compliance officer en las Administraciones públicas.

A diferencia lo de expuesto en relación con las empresas o cualquier otra persona jurídica de derecho privado -asociaciones, sindicatos, partidos políticos-, por el contrario, el escenario del control y supervisión en el marco de las administraciones públicas es un proceso regulado, no de autorregulación. Es un proceso de estricta regulación legal. De ahí también que, correctamente, el legislador penal haya dejado fuera de la responsabilidad penal al Estado y a las administraciones públicas territoriales e institucionales, a los organismos reguladores, a las agencias y entidades públicas empresariales, a las organizaciones internacionales de derecho público y a todas aquellas que ejerzan potestades públicas de soberanía o administración. Está claro la causa por la que el legislador las excluye como sujetos del Derecho penal: precisamente, porque su actuación está determinada por el «ejercicio de funciones públicas de soberanía o administración» (art. 31 quinquies $\mathrm{CP}$ ).

De ahí que resulte, cuando menos extraño que se plantee en la idea de compliance -entendido como «autorregulación» de la organización empresarial para la prevención, supervisión y control de riesgos penales- en las administraciones públicas. En la Administración pública todos los mecanismos de control y supervisión de la actividad se encuentran regulados por los procesos establecidos en la ley (Ley 40/2015, de 1 de octubre, de Régimen Jurídico del Sector Público), así como los concretos pasos y procesos de la contratación pública (Ley 39/2015, de Contratos del Sector Público). No cabe hablar en este contexto de «autorregulación». La Administración del Estado y las administraciones públicas son regulación en el más estricto sentido.

Si lo que se trata es de prevenir la corrupción en la contratación pública, habrá que revisar y, en su caso, reformar las normas que establecen las «funciones de control y supervisión», los "conflictos de intereses», entre otros, en dicho proceso de contratación.

La reforma de la Ley 9/2017, de 8 de noviembre, de Contratos del Sector público, por la que se incorporó a la legislación española las Directivas 2014/23/UE y 
2014/24/UE, ha llevado a cabo una importante revisión de nuestra legislación en esta materia. Como señala la propia Exposición de Motivos los objetivos que inspiran la regulación contenida en la nueva Ley son, en primer lugar, lograr una mayor «transparencia en la contratación pública»-rendición de cuentas-, y, en segundo lugar, conseguir una «mejor relación calidad-precio»-libre concurrencia-. Para lograr este último objetivo por primera vez se establece la obligación de los órganos de contratación de velar porque el diseño de los criterios de adjudicación permita obtener obras, suministros y servicios de gran calidad, concretamente mediante la inclusión de aspectos cualitativos, medioambientales, sociales e innovadores vinculados al objeto del contrato. Estas medidas, son sin duda medidas para mejorar la contratación pública, pero además son medidas de prevención y, en este sentido, es compliance.

Se puede decir que la Ley ya preveía y prevé ahora de forma mejorada muchas «funciones de compliance», si con ello nos queremos referir a las funciones de control y supervisión que implican funciones de garante. Si aún resultan deficientes para la prevención de la corrupción, deberán ser reformadas.

Si nos fijamos de forma muy resumida en un proceso de contratación de obras, concesión de obras, concesión de servicios, suministro o de servicios, se puede decir que el esquema general responde a tres pasos importantes a efectos de las funciones de control: a) elaboración de los pliegos de prescripciones generales y técnicas (PSA/PPT), b) valoración de la mesa de contratación y c) recepción del servicio o mercancía en conformidad por parte del órgano de contratación. En estas tres fases es donde habrá que revisar muy especialmente si están previstas todas las medidas necesarias de prevención de la corrupción. Tanto en el momento en que el órgano de contratación define los términos del contrato y requisitos, así como los requisitos de solvencia del prestatario, el elemento esencial de control es determinar la «necesidad» del servicio e idoneidad del contrato (art. 28 LCSP). Los pliegos pueden, según los casos, requerir un informe previo de la asesoría jurídica que puede ser o no preceptivo. Pero en todo caso, si hay un elemento importante a valorar por la asesoría jurídica es la concurrencia de la «necesidad y eficiencia» de la contratación propuesta. Por otro lado, en el momento en que la mesa de contratación valora las ofertas y el cumplimiento de los requisitos, valora si las propuestas se ajustan a los criterios de valoración, es un segundo momento de especial importancia. No hay que olvidar que en la mesa de contratación hay tanto un miembro de la intervención, como de la asesoría jurídica. Estas dos deben velar por el cumplimiento legal y, por tanto, son «funciones de compliance». También se lleva a cabo una «función de compliance» cuando el órgano de contratación recibe en conformidad el servicio o mercancía. Por último, la fiscalización del gasto por parte de la intervención es también otro escalón de control y supervisión. Dicho con otras palabras: todo control y supervisión de la legalidad son momentos en los que se están llevando a cabo «funciones de compliance». Estas funciones son realizadas por las diversas instituciones que ostentan las funciones de supervisión o de control, así, la Intervención General del Estado, la asesoría jurídica/Abogacía del Estado, el Tribunal de Cuentas.

Además de las funciones y deberes de control y supervisión en estas tres fases se presenta, desde un punto de vista de la prevención de la corrupción, un aspecto esencial: es una cuestión nuclear que estén correcta y expresamente regulados los supuestos de conflictos de intereses, en los que puedan incurrir las personas que deban cumplir con estas funciones. Es, sin duda, el aspecto más espinoso en el ámbito de la prevención de la corrupción en la contratación pública.

Todas las «funciones de control y supervisión», por otro lado, definen no solo las competencias de los respectivos órganos, sino también las posiciones de garante 
que, a efectos de la eventual responsabilidad penal de los funcionarios, son funciones que definen la posición de garante de los funcionarios (art. $11 \mathrm{CP}$ ). Es decir, el incumplimiento de los deberes legales de control y supervisión pueden dar lugar a la comisión de delitos de prevaricación -administrativa- y/o malversación, delito de tráfico de influencias, de fraudes y exacciones ilegales, de negociaciones y actividades prohibidas a los funcionarios, entre otros delitos de funcionarios.

En conclusión, la idea de una nueva figura de un public compliance officer (Queralt, 2016, pp. 9 y ss.) en la Administración pública resulta no solo complejo, sino difícil de conciliar con los deberes propios de la Administración y, sobre todo, casi imposible de diferenciar de las funciones propias que por ley ejercen la Intervención General y control financiero y la asesoría jurídica en el marco de los órganos de la contratación pública.

En este sentido, no parece que se trata tanto de la necesidad de introducir la nueva figura del compliance officer-pensada para el ámbito de la autorregulación en el sector privado-, sino de definir adecuadamente los «deberes de control y supervisión» en el desarrollo del proceso de contratación. En esta revisión de los procesos de contratación, los «programas de compliance»-que reposan sobre cuatro pilares esenciales: prevención, detección, reacción, seguimiento- pueden ofrecer un «método» o un «modelo» muy apropiado para revisar la legislación existente y revisar los riesgos existentes ${ }^{11}$. La Ley de Contratación del Sector Público (Ley 9/2017) ha llevado a cabo ya una importante revisión de estos procesos, pero aún es una tarea inconclusa.

Cuestión distinta, son las «sociedades mercantiles públicas que ejecutan políticas públicas o prestan servicios de interés económico general». El Código penal dispone en su art. 31 quinquies 2, que son penalmente responsables -como toda empresa privada en el sentido de lo dispuesto en el art. 31 bis y ss.- pero establece una «limitación» en cuanto a las penas que se les puede aplicar: solo pueden ser sancionadas con la pena de multa (art. 33.7 a) CP) o con la pena de intervención judicial para salvaguardar los derechos de los trabajadores o acreedores (art. $33.7 \mathrm{~g}$ ) $\mathrm{CP}$ ). Esta limitación tiene plena justificación: sería impensable imponer las penas de prohibición de actividades o disolución, por ejemplo, en empresas públicas que ejecutan políticas públicas y/o prestan servicios de interés económico general. En este sentido, no cabe duda alguna en la doctrina jurídico penal que las sociedades mercantiles empresariales responden en el mismo sentido que cualquier otra sociedad mercantil y les es, por tanto, aplicable todo lo dicho al respecto. Las sociedades mercantiles públicas están sometidas a regulación especial en cuanto a ciertos controles y supervisión de su actividad, así como en relación con la composición de su órgano de administración, pero en su actividad empresarial los contratos de prestación de servicios se rigen por el derecho privado.

\section{Conclusiones y resumen}

Compliance representa hoy en día, sin duda, un ámbito de estudio jurídico nuevo que se refiere a la prevención de riesgos de la actividad empresarial que van desde los propios del Derecho mercantil y del Derecho privado hasta los del Derecho penal, pasando por los del Derecho administrativo. Desde esta perspectiva es un objeto de estudio transversal.

\footnotetext{
${ }^{11}$ Queralt, J. J. (2016, p. 5 y ss.) hace una propuesta de definición de las funciones nucleares de un public compliance officer. Véase extensamente Turienzo Fernández, A. (2021, pp. 43 y ss.).
} 
En la práctica ha adquirido una mayor importancia el compliance penal, como mecanismo para la prevención de riesgos de delitos previstos para las personas jurídicas. En este sentido, constituye un ámbito específico y a la vez limitado propio del Derecho penal económico. Se podría decir que al igual que el Derecho penal representa el máxima grado de responsabilidad que le puede ser atribuido a un sujeto, el compliance penal representa la prevención y reducción los riesgos más graves de la actividad empresarial regulados en el ordenamiento jurídico.

En la jurisprudencia del Tribunal Supremo ya se han dictado importantes sentencias en cuanto a la responsabilidad penal de las personas jurídicas y a los criterios de imputación, pero aún no se han dictado sentencias que hayan evaluado el efectivo funcionamiento de un «programa de compliance».

Quedan aún muchas cuestiones jurídicas abiertas y de estudio en torno al significado y alcance del compliance. Es un ámbito que se encuentra en evolución, pero al menos se puede decir que es una pieza esencial de la organización empresarial para la prevención y disminución de los riesgos inherentes a la actividad empresarial y para la responsabilidad penal de las personas jurídicas.

Bibliografía

Aguilera Gordillo, R. (2018). Compliance Penal en España. Pamplona, España: Aranzadi.

Alfaro, J. (26 abril, 2016). Cultura empresarial y gobierno corporativo. Recuperado 5 de septiembre de 2021, de https://almacendederecho.org/cultura-empresarial-ygobierno-corporativo.

Bacigalupo, E. (2011). Compliance y Derecho penal. Pamplona, España: Aranzadi.

Bacigalupo, S. (2021). La protección de los alertadores desde la perspectiva jurídicopenal. Breves reflexiones sobre la transposición de la Directiva 2019/1937. En J. Ponce Solé, M. Villoria Mendieta, y O. Capdeferro Villagrasa (eds.), Anuario del Buen Gobierno y de la Calidad de la Regulación ABGCR/2020 (1.a ed., pp. 163181). Madrid, España: Fundación Democracia y Gobierno Local.

Bacigalupo, S. (2020). Posición de garante del órgano de control y supervisión de riesgos penales en el ámbito societario. Diario La Ley, 9632.

Bacigalupo, S. (1998). Responsabilidad Penal de las Personas Jurídicas. Barcelona, España: Bosch.

Bock, D. (2011). Criminal Compliance. Baden-Baden, Alemania: Nomos Verlagsgesellschaft.

Bock, D. (2009). Strafrechtliche Aspekte der Compliance-Diskussion - $§ 130$ OWiG als zentrale Norm der Criminal Compliance. Zeitschrift für Internationale Strafrechtsdogmatik (ZIS), 2(2009), pp. 68-81.

Grennan, J. (2019). A Corporate Culture Channel: How Increased Shareholder Governance Reduces Firm Value. SSRN. https://doi.org/10.2139/ssrn.2345384.

Hauschka, C., Moosmayer, K., y Lösler, T. (Eds.). (2010). Corporate Compliance: Handbuch der Haftungsvermeidung im Unternehmen (2.a ed.). Munich, Alemania: C.H.Beck.

Hauschka, C., y Greeve, G. (2007). Compliance in der Korruptionsprävention was müssen, was sollen, was können die Unternehmen tun? Betriebs-Berater (BB), 62(4), pp. 165-173.

Kreps, D. (1990). Corporate Culture and Economic Theory. Perspectives on Positive Political Economy. Cambridge University Press, pp. 90-142. https://doi.org/10.1017/CBO9780511571657.006.

Kuhlen, L., Kudlich, H., y Ortiz De Urbina, I. (eds.) (2013). Compliance und Strafrecht. Heidelberg, Alemania: Verlagsgruppe Huthig Jehle Rehm. 
Kuhlen, L. (2009). Strafrechtliche Haftung von Führungskräften. En F. Maschmann (ed.), Corporate Compliance und Arbeitsrecht (1.a ed., pp. 11-30). Baden-Baden, Alemania: Nomos Verlagsgesellschaft. https://doi.org/10.5771/978384521881611.

Lin, T. (2017). Compliance, Technology, and Modern Finance. Brooklyn Journal of Corporate, Financial y Commercial Law, 11, pp. 159-182.

Luhmann, N. (1995). Das Recht der Gesellschaft. Frankfurt am Main, Alemania: Suhrkamp Verlag.

Queralt, J. J. (2016). Public compliance y corrupción: análisis conceptual y propuestas. Revista Internacional de Transparencia e Integridad (R.I.T.I), 2, pp. $1-11$.

Rotsch, T. (2012). Criminal Compliance. InDret, 1(2012), pp. 1-11.

Ruiz De Lara, M., Gollonet Teruel, L. A., Pérez-Piaya Moreno, C., y Serrano Zaragoza, O. (eds.). (2018). Compliance penal y responsabilidad civil y societaria de los administradores (1.a ed.). Barcelona, España: Bosch.

Schein, E. H. (1986). Organizational Culture and Leadership. The Academy of Management Review, 11(3), pp. 677-680. https://doi.org/10.2307/258322.

Turienzo Fernández, A. (2021). La responsabilidad penal del compliance officer. Madrid, España: Marcial Pons. 